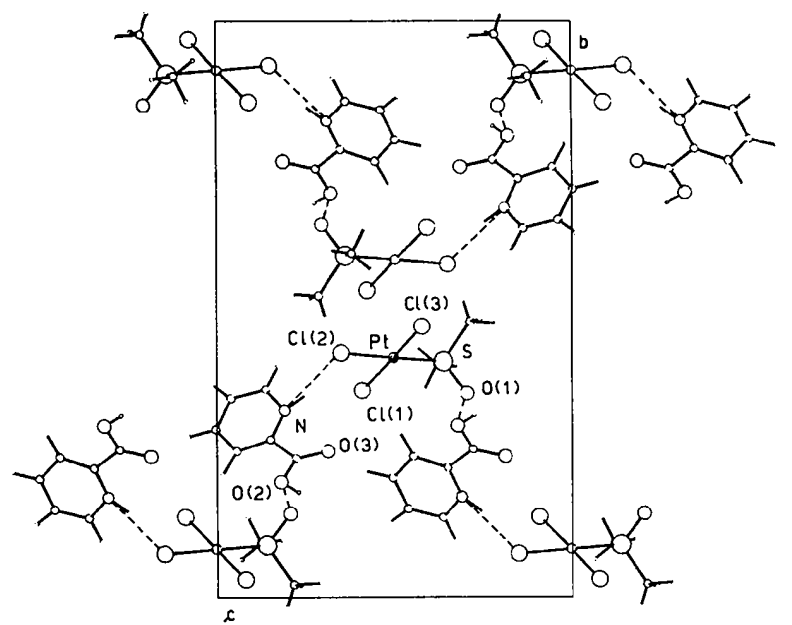

Fig. 2. Projection along [100] of the packing of $\left[\mathrm{H}_{2} \mathrm{pic}\right]\left[\mathrm{PtCl}_{3}\left(\mathrm{Me}_{2} \mathrm{SO}\right)\right]$.

displaced from the mean plane passing through them by $-0.003(2), 0.029(4),-0.009(2), 0.030(4)$ and -0.008 (2) $\AA$, respectively. The structural parameters of $\left[\mathrm{PtCl}_{3}\left(\mathrm{Me}_{2} \mathrm{SO}\right)\right]^{-}$are quite comparable to those of the same complex in the structure of the potassium salt (2) (Melanson, Hubert \& Rochon, 1976) in which the values of the $\mathrm{Pt}-\mathrm{Cl}$ bonds were
$2 \cdot 302(6), 2 \cdot 318(5)$ and $2 \cdot 296(6) \AA$, and the Pt-S bond $2 \cdot 193(5) \AA$.

In contrast, some differences in the IR spectra of (1) and (2) have been found. (i) The stretching $\mathrm{S}-\mathrm{O}$ falls at a lower frequency in (1) with respect to (2) $\left[\nu_{\mathrm{S}=0}: 1060 \mathrm{~cm}^{-1}\right.$ (1), $1100 \mathrm{~cm}^{-1}$ (2)]; (ii) The stretching $\mathrm{Pt}-\mathrm{Cl}$ (Cl trans to sulfoxide) decreases from $309 \mathrm{~cm}^{-1}$ (1) to $290 \mathrm{~cm}^{-1}$ (2), probably due to the weakness of the bond determined by the hydrogen bond with the 2-carboxypyridinium cation.

The packing of the structure, shown in Fig. 2, is determined by an intermolecular hydrogen bond involving the $-\mathrm{OH}$ group of the pyridine-2carboxylic acid moiety and the $\mathrm{O}$ atom of the dimethyl sulfoxide moiety $\left[\mathrm{O}(2) \cdots \mathrm{O}(1)\left(x+\frac{1}{2}, \frac{1}{2}-y\right.\right.$, $1-z)=2 \cdot 570(12) \AA, \mathrm{O}(2)-\mathrm{H}(2)-\mathrm{O}(1)\left(x+\frac{1}{2}, \frac{1}{2}-y\right.$, $\left.1-z)=163^{\circ}\right]$.

\section{References}

Annibale, G., Cattalnini L., Canovese, L., Pitteri, B., TiripicChio, A., Tiripicchio Camellin, M. \& Tobe, M. L. (1986). J. Chem. Soc. Dalton Trans. pp. 1101-1105.

Melanson, R., Hubert, J. \& Rochon, F. D. (1976). Acta Cryst. A32, 1914-1916.

SHELDRICK, G. M. (1976). SHELX76. Program for crystal structure determination. Univ. of Cambridge, England.

Ugozzoli, F. (1987). Comput. Chem. 11, 109-120.

Walker, N. \& Stuart, D. (1983). Acta Cryst. A39, 158-166.

Acta Cryst. (1991). C47, 703-705

\title{
Structure of Dichlorobis(p-phenoxyphenyl)tellurium(IV)
}

\author{
By M. De Matheus and L. TORRES \\ Departamento de Quimica, Universidad Nacional de Columbia, Bogotá, Colombia \\ J. F. Piniella* and J. L. Briansó \\ Area de Cristalografía, Universidad Autónoma de Barcelona, 08193 Bellaterra, Spain
}

and C. Miravitlles

Instituto de Ciencia de Materials (CSIC), c/Martí i Franquès, s/n, 08028 Barcelona, Spain

(Received 20 March 1990; accepted 28 June 1990)

Abstract. $\left[\mathrm{TeCl}_{2}\left(\mathrm{C}_{12} \mathrm{H}_{9} \mathrm{O}\right)_{2}\right], M_{r}=536.9$, monoclinic, $P 2_{1} / c, a=8.061(5), b=21.694(5), c=12.606(7) \AA$, $\beta=101.5(2)^{\circ}, \quad V=2160(2) \AA^{3}, \quad Z=4, \quad D_{x}=$ $1.652 \mathrm{~cm}^{-3}, \quad$ Mo $K \alpha, \quad \lambda=0.701069 \AA, \quad \mu=$ $16.45 \mathrm{~cm}^{-1}, F(000)=1056$, room temperature, $R=$ $0.028, w R=0.030$ for 2626 observed reflections $[I \geq$ $2 \cdot 5 \sigma(I)]$. The Te atom is in a pseudo trigonal-

* To whom correspondence should be addressed. 0108-2701/91/040703-03\$03.00 bipyramidal configuration with the lone pair in one of the equatorial positions. Distances $\mathrm{Te}$ $\mathrm{Cl}=2.506(1), \quad 2.510$ (1) $\AA ; \quad \mathrm{Te}-\mathrm{C}=2 \cdot 107$ (3), $2 \cdot 129$ (4) $\AA ; \mathrm{Cl}-\mathrm{Te}-\mathrm{Cl}=177 \cdot 21(1)^{\circ} ; \mathrm{Cl}-\mathrm{Te}-\mathrm{C}=$ $90.7(1), 89.2(1), 88.6(1), 88.7(1)^{\circ} ; \mathrm{C}-\mathrm{Te}-\mathrm{C}=$ $98 \cdot 7(1)^{\circ}$.

Introduction. Diorganyl ditellurides and diorganyl tellurides constitute an important class of starting (C) 1991 International Union of Crystallography 
materials for the preparation of other organotellurium compounds (Petragnani \& Comasseto, 1986). The well known synthetic route whereby diaryl ditellurides, arenetellurenyl halides (generated in situ) are treated with Grignard reagents to produce unsymmetrical aryl alkyl tellurides (Petragnani, Torres \& Wynne, 1975) has been used as a general method for preparing other organotellurium compounds (Dabdoub, Dabdoub, Comasseto \& Petragnani, 1986). Because of the increasing interest in the field of organic chemistry, a series of dihalobis(aryl)tellurium(IV) compounds were prepared following the procedure described by Drew (1926). A structural study of the title compound was undertaken in order to examine the bonding and molecular geometry.

Experimental. Prismatic red-brown crystals suitable for X-ray study $(0.16 \times 0.08 \times 0.03 \mathrm{~mm})$, EnrafNonius CAD-4 diffractometer, using the 'flat $\psi$ mode' to minimize absorption. Unit-cell parameters by least squares from 25 reflections with $\theta$ between 6 and $13^{\circ} ; 3192$ unique reflections collected, 2626 considered observed with $I \geq 2 \cdot 5 \sigma(I) ; \omega-2 \theta$ scan mode, $h k l$ range: $-8 \leq h \leq 8,-0 \leq k \leq 23,0 \leq l \leq 14, \theta$ limit $25^{\circ}$; scan range $\Delta \theta=(0.80+0.34 \tan \theta)^{\circ}$; no significant decay from monitoring of three standard reflections. Lorentz-polarization corrections, no absorption correction. Structure solved by heavyatom methods (SHELX76; Sheldrick, 1976). Te atom located from Patterson functions, remaining atoms (except H9) by subsequent Fourier maps. Anisotropic full-matrix least-squares refinement on $F$ of non-H atoms (SHELX76). H9 position was calculated for $\mathrm{C}-\mathrm{H}=0.96 \AA$; isotropic temperaturefactor refinement for $\mathrm{H}$ atoms $\left(U=0.0836 \AA^{2}\right) ; 314$ parameters refined, $R=0.028, w R=0.030, w=$ $0.7213 /\left[\sigma^{2}(F)+0.00109 F^{2}\right] ; \quad \max . \quad(\Delta / \sigma)=0.064$. Residual electron density within -0.94 and $0.47 \mathrm{e} \AA^{-3}$ near the Te atom. Scattering factors (except tellurium) from International Tables for $X$-ray Crystallography (1974, Vol. IV); scattering factors for tellurium from Forsyth \& Wells (1959). Drawing by PLUTO (Motherwell \& Clegg, 1978). All calculations were performed on a Digital MicroVAX II.

Discussion. The atomic parameters are given in Table $1 .^{*}$ A view of the molecule with the atomic numbering scheme is shown in Fig. 1 , and the molecular packing in Fig. 2. Table 2 gives bond distances and angles.

\footnotetext{
* Lists of structure factors, anisotropic thermal parameters, $\mathrm{H}$-atom coordinates and least-squares planes have been deposited with the British Library Document Supply Centre as Supplementary Publication No. SUP 53353 (21 pp.). Copies may be obtained through The Technical Editor, International Union of Crystallography, 5 Abbey Square, Chester CH1 2HU, England.
}

Table 1. Atomic coordinates $\left(\times 10^{4}, \times 10^{5}\right.$ for $\left.\mathrm{Te}\right)$ with e.s.d.'s in parentheses and equivalent isotropic temperature factors $\left(\AA^{2}\right)$ for non-H atoms

\begin{tabular}{|c|c|c|c|c|}
\hline & $B_{\mathrm{eq}}$ & $\left.{ }^{2} / 3\right) \sum_{i} \sum_{j} U_{i j}$ & ${ }^{*} \mathbf{a}_{i} \cdot \mathbf{a}_{j}$ & \\
\hline & $x$ & $y$ & $z$ & $B_{\text {eq }}$ \\
\hline $\mathrm{Te}$ & $87768(3)$ & $6455(1)$ & $63321(2)$ & 3.64 \\
\hline $\mathrm{Cll}$ & $9647(1)$ & 1530 (1) & 7599 (1) & $5 \cdot 43$ \\
\hline $\mathrm{Cl}$ & $6759(5)$ & $475(2)$ & $7127(3)$ & 3.66 \\
\hline $\mathrm{Cl} 2$ & 7772 (1) & -243 (1) & 5095 (1) & $4 \cdot 76$ \\
\hline $\mathrm{C} 2$ & $6712(6)$ & $-85(2)$ & 7625 (3) & $4 \cdot$ \\
\hline C3 & $5374(7)$ & $-203(2)$ & 8170 (4) & $5 \cdot 8$ \\
\hline $\mathrm{C4}$ & $4173(5)$ & 241 (2) & 8192 (3) & $4 \cdot 5$ \\
\hline $\mathrm{C} 5$ & $4254(6)$ & $794(2)$ & $7706(4)$ & $5 \cdot 0$ \\
\hline C6 & $5548(5)$ & $925(2)$ & 7168 (3) & $4 \cdot 63$ \\
\hline $\mathrm{O} 1$ & $2910(5)$ & $154(2)$ & 8776 (3) & $7 \cdot 4$ \\
\hline $\mathrm{C} 7$ & $1900(6)$ & $-374(2)$ & 8590 (4) & $4 \cdot 8$ \\
\hline $\mathrm{C} 8$ & $1325(7)$ & $-609(3)$ & $9461(4)$ & $6 \cdot 1$ \\
\hline $\mathrm{C} 9$ & $269(7)$ & $-1111(3)$ & $9325(4)$ & $6 \cdot 8$ \\
\hline $\mathrm{C} 10$ & $-188(6)$ & $-1380(3)$ & $8336(5)$ & \\
\hline $\mathrm{Cll}$ & $390(6)$ & $-1144(3)$ & $7471(4)$ & $5 \cdot 8$ \\
\hline $\mathrm{Cl} 2$ & $1444(6)$ & $-638(2)$ & 7595 (4) & 4.5 \\
\hline $\mathrm{C} 13$ & $7540(5)$ & $1280(2)$ & $5144(3)$ & 3.7 \\
\hline $\mathrm{Cl} 4$ & $6053(5)$ & $1126(2)$ & 4460 (3) & $4 \cdot 1$ \\
\hline $\mathrm{Cl} 5$ & $5315(5)$ & $1534(2)$ & 3666 (3) & $4 \cdot$ \\
\hline $\mathrm{C} 16$ & $6111(5)$ & $2092(2)$ & $2556(3)$ & $4 \cdot 6$ \\
\hline $\mathrm{Cl} 7$ & $7573(6)$ & $2244(2)$ & 4239 (4) & $5 \cdot$ \\
\hline $\mathrm{C} 18$ & $8290(6)$ & $1839(2)$ & 5047 (3) & \\
\hline $\mathrm{O} 2$ & $5404(5)$ & 2529 (1) & $2795(2)$ & $6 \cdot 1$ \\
\hline $\mathrm{C} 19$ & 4692 (5) & $2333(2)$ & 1751 (3) & $4 \cdot 4$ \\
\hline $\mathrm{C} 20$ & $3274(6)$ & $2641(2)$ & 1224 (4) & $5 \cdot 5$ \\
\hline $\mathrm{C} 21$ & $2617(6)$ & 2490 (3) & $157(4)$ & \\
\hline $\mathrm{C} 22$ & $3320(6)$ & $2036(3)$ & $-354(4)$ & \\
\hline $\mathrm{C} 23$ & $4735(7)$ & 1741 (3) & $176(4)$ & $6 \cdot 1$ \\
\hline C24 & $5439(6)$ & 1894 (2) & $1233(3)$ & $5 \cdot 52$ \\
\hline
\end{tabular}

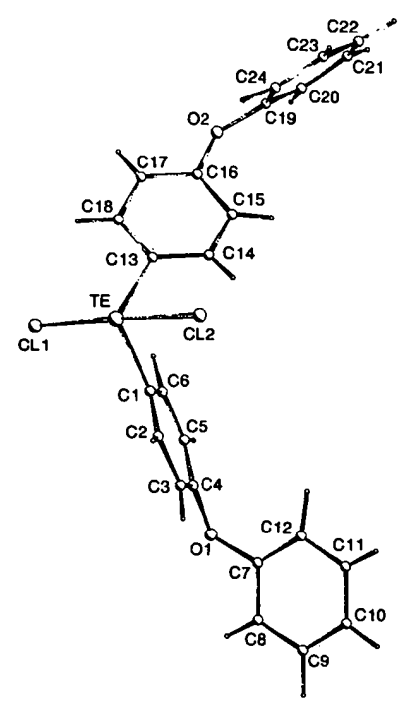

Fig. 1. A view of the molecule showing the atomic numbering.

The bonding in the molecule is consistent with the predictions of classical bonding models for $A B_{4} E$ molecules (Gillespie, 1970). $\mathrm{Cl}$ atoms are axial with a $\mathrm{Cl}-\mathrm{Te}-\mathrm{Cl}$ angle of $172 \cdot 2^{\circ}$ whereas two $\mathrm{C}$ atoms are placed in equatorial positions. The $\mathrm{Te}^{\mathrm{IV}}$ lone pair of electrons possibly occupies the vacant equatorial 
Table 2. Interatomic distances $(\AA)$ and angles $\left(^{\circ}\right)$ with e.s.d.'s in parentheses

\begin{tabular}{|c|c|c|c|}
\hline $\mathrm{Cl} 1-\mathrm{Te}$ & $2 \cdot 506(1)$ & $\mathrm{Cl1}-\mathrm{Cl0}$ & $1 \cdot 368(7)$ \\
\hline $\mathrm{Cl}-\mathrm{Te}$ & $2 \cdot 107(3)$ & $\mathrm{Cl} 2-\mathrm{Cll}$ & $1.376(7)$ \\
\hline $\mathrm{Cl} 2-\mathrm{Te}$ & $2 \cdot 510(1)$ & $\mathrm{Cl} 14-\mathrm{Cl3}$ & $1 \cdot 370(5)$ \\
\hline $\mathrm{Cl} 3-\mathrm{Te}$ & $2 \cdot 129(4)$ & $\mathrm{C} 18-\mathrm{C} 13$ & $1 \cdot 372(6)$ \\
\hline $\mathrm{C} 2-\mathrm{Cl}$ & $1 \cdot 370(5)$ & $\mathrm{Cl} 5-\mathrm{Cl} 4$ & $1 \cdot 379(6)$ \\
\hline $\mathrm{C} 6-\mathrm{Cl}$ & $1 \cdot 390(5)$ & $\mathrm{Cl} 16-\mathrm{Cl} 5$ & $1.391(6)$ \\
\hline $\mathrm{C} 3-\mathrm{C} 2$ & $1.414(6)$ & $\mathrm{Cl} 1-\mathrm{Cl} 6$ & $1 \cdot 354(5)$ \\
\hline $\mathrm{C} 4-\mathrm{C} 3$ & $1 \cdot 370(6)$ & $\mathrm{O} 2-\mathrm{C} 16$ & $1.386(5)$ \\
\hline $\mathrm{C} 5-\mathrm{C} 4$ & $1 \cdot 353(6)$ & $\mathrm{C} 18-\mathrm{C} 17$ & $1 \cdot 382(6)$ \\
\hline $\mathrm{O} 1-\mathrm{C} 4$ & $1 \cdot 383(5)$ & $\mathrm{C} 19-\mathrm{O} 2$ & $1.393(5)$ \\
\hline $\mathrm{C} 6-\mathrm{C} 5$ & $1 \cdot 383(6)$ & $\mathrm{C} 20-\mathrm{C} 19$ & $1 \cdot 375(6)$ \\
\hline $\mathrm{C} 7-\mathrm{O} 1$ & $1 \cdot 398(5)$ & $\mathrm{C} 24-\mathrm{C} 19$ & $1 \cdot 361(6)$ \\
\hline $\mathrm{C} 8-\mathrm{C} 7$ & $1 \cdot 373(6)$ & $\mathrm{C} 21-\mathrm{C} 20$ & $1 \cdot 383(7)$ \\
\hline $\mathrm{C} 12-\mathrm{C} 7$ & $1 \cdot 362(6)$ & $\mathrm{C} 22-\mathrm{C} 21$ & $1 \cdot 360(7)$ \\
\hline $\mathrm{C} 9-\mathrm{C} 8$ & $1 \cdot 373(7)$ & $\mathrm{C} 23-\mathrm{C} 22$ & $1 \cdot 361(7)$ \\
\hline $\mathrm{C} 10-\mathrm{C} 9$ & $1 \cdot 358(7)$ & $\mathrm{C} 24-\mathrm{C} 23$ & $1 \cdot 380(6)$ \\
\hline $\mathrm{Cl}-\mathrm{Te}-\mathrm{Cll}$ & $88 \cdot 6(1)$ & $\mathrm{Cl}-\mathrm{ClO}-\mathrm{C} 9$ & $119 \cdot 8(5)$ \\
\hline $\mathrm{Cl} 2-\mathrm{Te}-\mathrm{Cll}$ & $177 \cdot 2(1)$ & $\mathrm{Cl} 2-\mathrm{C} 11-\mathrm{Cl0}$ & $120 \cdot 7(5)$ \\
\hline $\mathrm{Cl} 2-\mathrm{Te}-\mathrm{Cl}$ & $88 \cdot 7$ (1) & $\mathrm{C} 11-\mathrm{Cl}-\mathrm{C} 7$ & $119 \cdot 1(4)$ \\
\hline $\mathrm{C} 13-\mathrm{Te}-\mathrm{Cl} 1$ & $89 \cdot 2(1)$ & $\mathrm{C} 14-\mathrm{C} 13-\mathrm{Te}$ & $120.9(3)$ \\
\hline $\mathrm{Cl} 3-\mathrm{Te}-\mathrm{Cl}$ & $98 \cdot 7(1)$ & $\mathrm{C} 18-\mathrm{C} 13-\mathrm{Te}$ & $118.8(3)$ \\
\hline $\mathrm{C} 13-\mathrm{Te}-\mathrm{Cl} 2$ & $90 \cdot 7(1)$ & $\mathrm{Cl} 1-\mathrm{Cl} 3-\mathrm{Cl} 4$ & $120 \cdot 3(4)$ \\
\hline $\mathrm{C} 2-\mathrm{C} 1-\mathrm{Te}$ & $117.9(3)$ & $\mathrm{Cl} 5-\mathrm{C} 14-\mathrm{Cl} 3$ & $119 \cdot 8(4)$ \\
\hline $\mathrm{C} 6-\mathrm{Cl}-\mathrm{Te}$ & $120.5(3)$ & $\mathrm{Cl}-\mathrm{Cl} 15-\mathrm{Cl} 4$ & $119 \cdot 4(4)$ \\
\hline $\mathrm{C} 6-\mathrm{Cl}-\mathrm{C} 2$ & $121 \cdot 5(3)$ & $\mathrm{Cl} 7-\mathrm{C} 16-\mathrm{Cl} 5$ & $120 \cdot 6(4)$ \\
\hline $\mathrm{C} 3-\mathrm{C} 2-\mathrm{Cl}$ & $118 \cdot 5(4)$ & $\mathrm{O} 2-\mathrm{Cl} 16-\mathrm{Cl} 5$ & $122 \cdot 0(4)$ \\
\hline $\mathrm{C} 4-\mathrm{C} 3-\mathrm{C} 2$ & $119.5(4)$ & $\mathrm{O} 2-\mathrm{Cl}-\mathrm{C} 17$ & $117 \cdot 3(4)$ \\
\hline $\mathrm{C} 5-\mathrm{C} 4-\mathrm{C} 3$ & $121 \cdot 0(4)$ & $\mathrm{Cl} 1-\mathrm{Cl}-\mathrm{Cl} 6$ & $119 \cdot 7(4)$ \\
\hline $\mathrm{O} 1-\mathrm{C} 4-\mathrm{C} 3$ & $120 \cdot 8(4)$ & $\mathrm{C} 17-\mathrm{C} 18-\mathrm{Cl} 3$ & $120 \cdot 1(4)$ \\
\hline $\mathrm{O} 1-\mathrm{C} 4-\mathrm{C} 5$ & $118 \cdot 0(4)$ & $\mathrm{C} 19-\mathrm{O} 2-\mathrm{Cl} 6$ & $118 \cdot 7(3)$ \\
\hline $\mathrm{C} 6-\mathrm{C} 5-\mathrm{C} 4$ & $120 \cdot 9(4)$ & $\mathrm{C} 20-\mathrm{C} 19-\mathrm{O} 2$ & $117 \cdot 1(4)$ \\
\hline $\mathrm{C} 5-\mathrm{C} 6-\mathrm{Cl}$ & $118 \cdot 5(4)$ & $\mathrm{C} 24-\mathrm{C} 19-\mathrm{O} 2$ & $121.9(4)$ \\
\hline $\mathrm{C} 7-\mathrm{Ol}-\mathrm{C} 4$ & $119 \cdot 2(3)$ & $\mathrm{C} 24-\mathrm{C} 19-\mathrm{C} 20$ & $120 \cdot 8(4)$ \\
\hline $\mathrm{C} 8-\mathrm{C} 7-\mathrm{O} 1$ & $116 \cdot 7(4)$ & $\mathrm{C} 21-\mathrm{C} 20-\mathrm{C} 19$ & $118.4(5)$ \\
\hline $\mathrm{C} 12-\mathrm{C} 7-\mathrm{O}$ & $122 \cdot 7(4)$ & $\mathrm{C} 22-\mathrm{C} 21-\mathrm{C} 20$ & $121 \cdot 1(5$ \\
\hline $\mathrm{C} 12-\mathrm{C} 7-\mathrm{C} 8$ & $120 \cdot 6(4)$ & $\mathrm{C} 23-\mathrm{C} 22-\mathrm{C} 21$ & $119 \cdot 7(4)$ \\
\hline $\mathrm{C} 9-\mathrm{C} 8-\mathrm{C} 7$ & $119.6(5)$ & $\mathrm{C} 24-\mathrm{C} 23-\mathrm{C} 22$ & $120 \cdot 2(5)$ \\
\hline $\mathrm{C} 10-\mathrm{C} 9-\mathrm{C} 8$ & $120 \cdot 2(5)$ & $\mathrm{C} 23-\mathrm{C} 24-\mathrm{C} 19$ & $119.7(4)$ \\
\hline
\end{tabular}

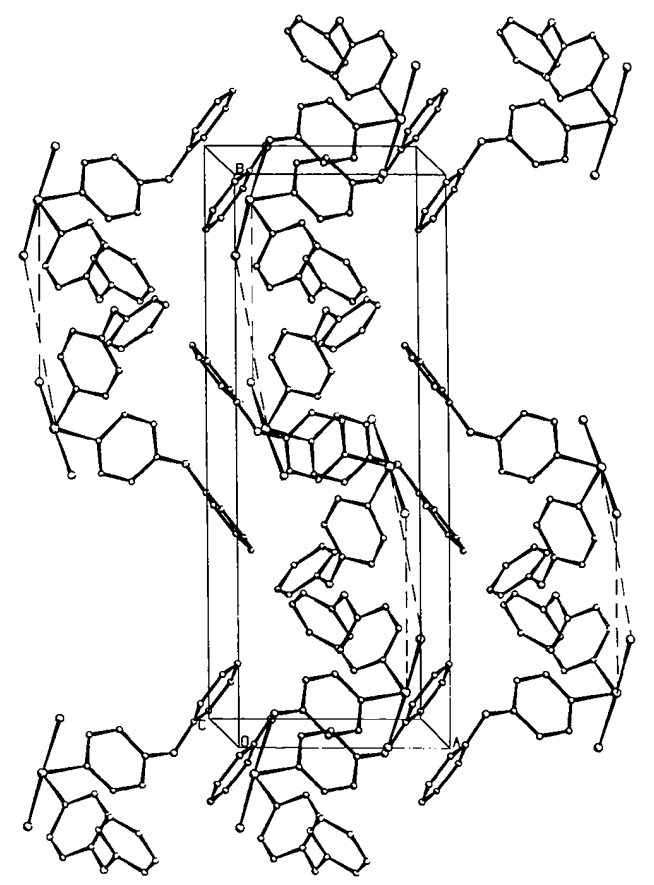

Fig. 2. Packing diagram, view down c. Intermolecular contacts are shown as dashed lines. position in the trigonal bipyramid as Ziolo and co-workers reported in their study on electron density for dimethyltellurium dichloride (Ziolo \& Troup, 1983). The $\mathrm{Te}-\mathrm{Cl}$ bond lengths are similar to those found in other $\mathrm{TeCl}_{2} R_{2}$ compounds, $2 \cdot 47-2.55 \AA$ (Alcock \& Harrison, 1982; Ziolo \& Troup, 1983; Husebye, Meyers, Zingaro, Comasseto \& Petragnani, 1987). The $\mathrm{Te}-\mathrm{C}$ bond distances are close to those predicted from the sum of the single-bond covalent radii, as in other $R_{2} \mathrm{TeCl}_{2}$ molecules (Chadha, Drake \& Kahn, 1983; Chada, Drake \& Hencher, 1983; Castellano, Zukerman-Schpector, Ferreira \& Comasseto, 1986). The $\mathrm{C}-\mathrm{Te}-\mathrm{C}$ and $\mathrm{Cl}-\mathrm{Te}-\mathrm{Cl}$ angles are also within the range reported for those compounds.

Intermolecular contacts are present: $\mathrm{Te}^{\cdots} \mathrm{Cl}^{\prime}{ }^{\prime}(2-$ $x,-y, 1-z) 3 \cdot 71 \AA$, and $\mathrm{Te}^{\cdots} \cdot \mathrm{C} 12^{\prime \prime}(x-1, y, z) 3 \cdot 68 \AA$ (shorter than the sum of respective van der Waals radii 3.81 and $3.83 \AA$ ), giving a distorted octahedral arrangement about the Te atom.

The phenyl rings are planar within experimental accuracy (r.m.s. deviation $=0.0044 \AA ; \delta_{\max }=$ $0.0073 \AA$ for C1, r.m.s. deviation $=0.0024 \AA, \delta_{\max }=$ $0.0037 \AA$ for C9, r.m.s. deviation $=0.0077, \delta_{\max }=$ 0.0105 for C15, r.m.s. deviation $=0.0102, \delta_{\max }=$ 0.0146 for $\mathrm{C} 21$ ). The bond lengths and angles in the organic moiety are within the expected range of values.

Financial support from the European Economic Community (M. de Matheus Research Fellowship) is gratefully acknowledged.

\section{References}

Alcock, W. \& Harrison, W. D. (1982). J. Chem. Soc. Dalton Trans. pp. 251-255.

Castellano, C., Zukerman-Schpector, J., Ferreira, J. T. \& Comasseto, J. V. (1986). Acta Cryst. C42, 44-46.

Chadha, R. K., Drake, J. E. \& Hencher, J. L. (1983). Can. J. Chem. pp. 1222-1225.

Chadha, R. K., Drake, J. E. \& Kahn, M. A. (1983). Acta Cryst. C39, 889-899.

Dabdoub, M. J., Dabdoub, V. B., Comasseto, J. V. \& Petragnani, N. (1986). J. Organomet. Chem. 308, 211-222.

Drew, H. D. K. (1926). J. Chem. Soc. pp. 223-232.

Forsyth, J. B. \& Wells, M. (1959). Acta Cryst. 12, 412-415.

Gillespie, R. J. (1970). J. Chem. Educ. 47, 18-23.

Husebye, S., Meyers, E. A., Zingaro, R. A., Comasseto, J. V. \& Petragnani, N. (1987). Acta Cryst. C43, 1147-1151.

Motherwell, W. D. S. \& ClegG, W. (1978). PLUTO78. Program for plotting molecular and crystal structures. Univ. of Cambridge, England.

Petragnani, N. \& Comasseto, J. V. (1986). Synthesis, 1, 1-30.

Petragnani, N., Torres, L. \& Wynne, K. J. (1975). J. Organomet. Chem. 92, 185-189.

SHELDRICK, G. M. (1976). SHELX76. Program for crystal structure determination. Univ. of Cambridge, England.

Ziolo, R. F. \& Troup, J. M. (1983). J. Am. Chem. Soc. 105, 229-235. 